\title{
SUCCESSFUL OUTCOME IN A CASE OF CRITICAL DIGITAL ISCHEMIA. CASE REPORT AND LITERATURE ANALYSIS
}

\author{
Cristina Pomirleanu ${ }^{1,2}$, Georgiana Strugariu ${ }^{1}$, Andreea Axinte ${ }^{1}$, Iulian Resmerita ${ }^{1}$, Codrina Ancuta ${ }^{1,2}$ \\ ${ }^{1}$ Rheumatology 2 Department, Clinical Rehabilitation Hospital, Iasi \\ ${ }^{2}$ Grigore T. Popa University of Medicine and Pharmacy, Iasi
}

\begin{abstract}
Critical digital ischemia (CDI) remains a distinct, serious complication of peripheral vasculopathy in systemic sclerosis, macrovascular as well as microvascular disease, abnormal vascular remodeling, vasoconstriction-vasodilation imbalance and fibrosis being key underlying factors. Successful short- as well as long-term outcomes may arise from early diagnosis, broad assessment and prompt therapeutic intervention. However, current treatment options are far from ideal, indicating the need for a complex approach focused on pathobiologic issues. We report the case of a middle age postmenopausal woman with a recent onset of CDI and subsequent gangrene, evolving in a very early PM-Scl positive systemic sclerosis. Dramatic improvement with complete resolution of CDI as well as healing of digital gangrene with minimal tissue loss was rapidly achieved under vasodilating therapy, after two cycles of intravenous prostanoids.
\end{abstract}

Keywords: critical digital ischemia, systemic sclerosis, anti-PM-Scl autoantibodies, prostanoids

\section{INTRODUCTION}

Peripheral vasculopathy, extensive and excessive skin and/or visceral fibrosis, inflammation and aberrant immune activation are key issues in the complex, multifaceted pathobiology of systemic sclerosis (SSc) $(1,2)$. Critical digital ischemia (CDI) defined as impaired perfusion with compromised tissue viability, is an uncommon, potentially severe complication of peripheral vasculopathy in SSc. Early diagnosis, complex assessment and prompt initiation of full vasodilating therapy are fundamental in order to prevent irreversible tissue damage with necrosis, gangrene and/or digital loss $(2,3)$. Despite a stratified and personalized approach based on recently emerging recommendations, the optimal therapeutic algorithm for CDI comprising vasoactive drugs, antiplatelet therapy, remissives, and, ultimately, surgical options, is still under debate (3).

We report the case of a middle age postmenopausal woman with a recent onset of extensive CDI complicated with gangrene, in a very early, PM-Scl positive SSc. Prompt intravenous prostacycline resulted in spectacular improvement with complete resolution of digital ischemia, healing of gangrene, with tissue loss limited to the distal part of the phalanx.
We further review recent advances in SSc associated CDI pathobiology and stratified therapeutic algorithm.

\section{CASE REPORT}

A 50 years old postmenopausal woman, with no significant familial or personal pathological history except a 10-months Raynaud's phenomenon (RP), was referred to the outpatient rheumatology department for a progressive digital ischemia with tissue necrosis of the upper limbs. Clinical evaluation showed CDI of the $3^{\text {rd }}$ and $4^{\text {th }}$ right and $2^{\text {nd }}$ and $3^{\text {rd }}$ left hands digits, with fingertip necrosis in the $3^{\text {rd }}$ and $4^{\text {th }}$ right digits, and frank gangrene in the distal phalanx of the $2^{\text {nd }}$ finger of the right hand, with visible line of demarcation, extremely painful and disabling (Fig. 1a). No other vascular lesions (e.g. digital ulcers or digital pitting scars), sclerodactyly or thickened skin were described, with normal peripheral pulses and ankle-brachial pressure index (ABPI). A complex assessment was considered to identify the underlining etiology of the CDI and gangrene in our RP-positive patient, supposing a SSc or non-SSc connective tissue disorder (with either proximal occlusive disease, vasculitis, thromboembolic disease, or severe

Correspondence address:

Codrina Ancuta, MD, Grigore T. Popa University of Medicine and Pharmacy, 16 Universitatii Street, Iasi, Romania

E-mail: codrina_ancuta@yahoo.com 
distal microvascular disease), a large vessel disease (e.g. Takayasu disease), thrombophilia (e.g. antiphospholipid syndrome), embolic disease (thrombotic and septic emboli), paraproteinaemia, or paraneoplastic phenomenon.

Laboratory analysis confirmed a positive antinuclear antibody test with anti-PM-Scl specificity, while anti-double stranded DNA, antiphospholipid antibodies (lupus anticoagulant, anticardiolipin, anti-beta2-glycoprotein I antibodies), routine evaluation of systemic vasculitis (pANCA, cANCA), cryoglobulins, $\mathrm{C} 3$ and $\mathrm{C} 4$ complement fractions, complete blood count, liver and kidney tests, $\mathrm{C}$ reactive protein and ESR were all in normal range or negative. Furthermore, chest and hand X-rays, as well as echocardiography were normal, while an active scleroderma pattern (moderate loss of capillaries, frequent giant capillaries and microhaemorrhages, mild ramified capillaries with mild architectural disorganization) was found on videocapillaroscopy (Fig. 1d).

We concluded that we are facing a SSc case fulfilling VEDOSS (Very Early Diagnosis of Systemic Sclerosis) criteria, a very early anti-PM-Scl positive disease with CDI (4). Since CDI and fingertip gangrene are acknowledged as a medical emergency, hospitalization was considered and aggressive treatment with intravenous Iloprost $(1 \mathrm{ng} / \mathrm{kg} / \mathrm{min})$ for 5 consecutive days and opioids were started, resulting in dramatic improvement with rapid limitation of necrotic tissue and pain intensity (Fig. 1b). No antico-

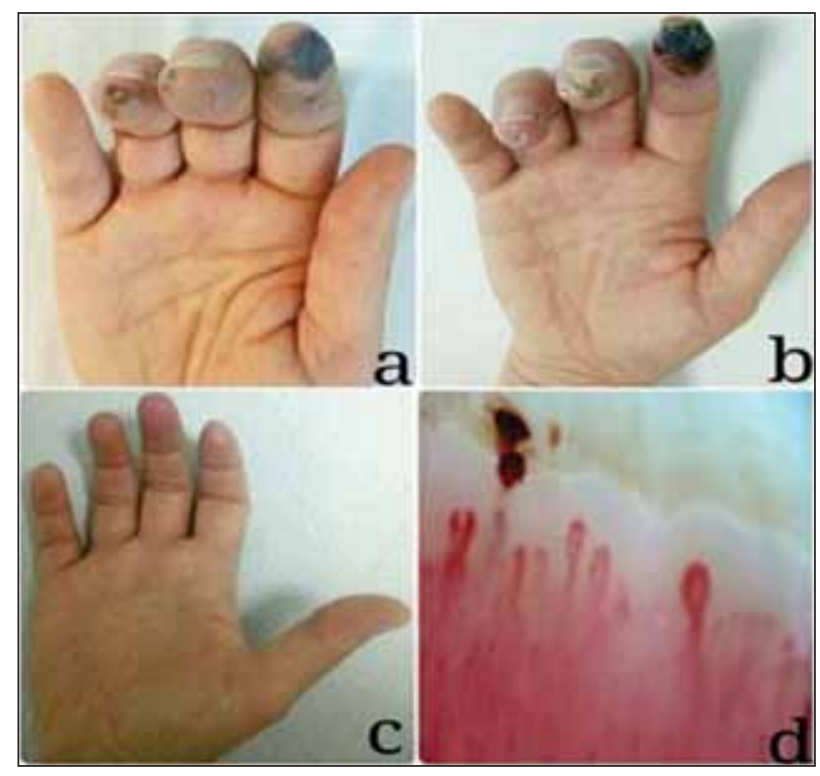

FIGURE 1. Critical digital ischemia

a. initial; b. after first infusion; c. after second infusion;

d. active scleroderma pattern agulation or antibiotics were required, while local debridement and digital sympathectomy were delayed according to the plastic surgeon recommendations. A second cycle of intravenous prostacycline was administered after one month, with concomitant antiplatelet agents, topical vasodilators (nitroglycerine 3\%) and Methotrexate (15mg weekly), prompting complete resolution of CDI, healing of the finger with gangrene, with autoamputation of the necrotic tissue and partial conservation of the involved distal phalanx (Fig. 1c).

\section{DISCUSSION}

We presented the challenging case of CDI with gangrene, the most severe expression of the digital vasculopathy spectrum in SSc, developing in a patient with very early (PM-Scl positive) disease.

It is widely recognized that CDI may occur in a large variety of pathologies, ranging from autoimmune disorders such as connective tissue diseases (SSc or non-SSc) and vasculitis, to diffuse arteriopathy, paraneoplastic syndromes, embolism, infection with septic shock, occupational diseases $(5,6)$. Defined by significant impairment of tissue perfusion, leading to intense and painful finger cyanosis, CDI secondary to SSc is typically associated with both macrovascular and microvascular disease, comprising narrowing of proximal and larger digital arteries, and intimal proliferation with luminal narrowing of small digital arteries, respectively (7). Additionally, fibrosis, dysregulated neuro-endothelial mechanism, an imbalance between vasoconstriction and vasodilation, abnormal vascular remodeling, platelet, and pro-coagulant status are major determinants of the multifaceted pathobiology of peripheral vasculopathy in SSc (7). Furthermore, another factor potentially contributing to vascular disease and digital ischemia might be atherosclerosis, although its particular role in SSc, with selective involvement of the ulnar artery and digital arteries is still controversial (8).

Several risk factors for CDI with or without subsequent (auto)amputation or digital loss are actually recognized, such as the diffuse subset of SSc (dc$\mathrm{SSc}$ ), a long history of digital vasculopathy, a precise pattern of antibodies (anti-Scl70, anti-centromere, anti-beta2-glycoprotein I and anti-granzyme B antibodies) and smoking status, as well (7). However, none of them was reported in our case: CDI and fingertip gangrene developed in a very early SSc in association with PM-Scl antibodies. Moreover, ANA 
positivity was not related here to either anti-topoisomerase, anti-centromere or anti-RNA polymerase III antibodies, but relied on anti-PM-Scl, targeting both PM-Scl-75 and PM-Scl-100 components (9). Not infrequently, dcSSc may associate anti-PMScl75 antibodies, with a clinical presentation of myositis-SSc overlap syndrome (10).

Urgent and complex clinical assessment and tailored management are essential in order to avoid complications related to CDI e.g. irreversible necrosis requiring amputation and digital loss (7).The algorithm for the evaluation of CDI in SSc includes several standard steps meaning clinical symptoms and signs assessment (cyanosis, pain, necrosis or gangrene), physical (peripheral and/or Doppler pulses, ABPI, Allen's test) and lab analysis (hypercoagulability work-up, anti-phospholipid and other autoantibodies, parameters for vasculitis) (7). Nailfold capillaroscopy, valuable for those patients presenting with CDI without a preexisting diagnosis of SSc, is very important, as well as routine cardiac assessment for embolic disease, with angiography performed in selected cases (7).

Both EULAR 2009 recommendations and the recent guideline formulated by the UK Systemic Sclerosis Study Group promote the early diagnosis as well as a personalized approach of peripheral vasculopathy (RP, digital ulcers, critical ischemia), focusing on vasoactive medication combined with antiplatelet therapy, remissive drugs, surgical debridement and/or sympathectomy $(7,11,12)$.

All SSc cases presenting with CDI require hospitalization for medical and/or surgical management. The sequential therapeutic practice is typically classified according to the presence or absence of necrosis; if uncomplicated, CDI benefits from intravenous vasodilator agents (intravenous prostanoids), analgesia, eventually anticoagulation, optimization of oral vasodilators $(7,12)$.

While successfully used for RP and digital ulcers, reducing the frequency and severity of RP and number of digital ulcers, respectively, prompt intravenous prostacyclin is also the standard therapy for uncomplicated CDI $(13,14)$. Despite being a potent vasodilator agent, Iloprost may also secondary inhibit platelet aggregation; however, the ideal dosing regimen has to be determined (7). Subsequently, oral vasodilator therapy including calcium channel blockers, angiotensin II receptor antagonists, alpha blockers, and phosphodiesterase type 5 inhibitors should be optimized in an attempt to maintain digital perfusion $(7,12,15,16)$. Newer vasodilators such as bosentan, an endothelin-1 receptor antagonist, may have a benefit in CDI linked to SSc, particularly for preventing the occurrence of new digital ulcers $(7,12,15,16)$. Finally, systemic anticoagulation, statin therapy and antiplatelet medication could also be administered, without full validation throughout clinical trials $(7,12,15-17)$. Opioid-based analgesia and appropriate antibiotics (either oral or IV) in infected ischemic lesions are commonly prescribed in routine practice, irrespective of the underlying pathobiology of CDI $(7,12,15,16)$.

In the particular case of necrotic complications or failure of medical therapies (persistent and/or progressive digital ischemic lesions), chemical and surgical sympathectomy, local administration of botulinum toxin, angioplastic or surgical vascular reconstruction, and/or surgical intervention (debridement and/or amputation) should be considered $(7,12$, 15,16).

Digital palmar sympathectomy must be considered only in severe extensive digital ischemia, as it helps promoting blood flow and preventing additional endothelial damage, without specific consensus on the exact extent of the intervention $(7,12$, $15,16,18)$.

Summarizing the therapeutic approach in our case, prompt intravenous Iloprost combined with systemic opioid analgesia had a successful and quite rapidly achieved outcome, with complete resolution of CDI and healing of the fingertip gangrene, with limited tissue loss, after the second cycle of prostacycline. Moreover, remissive therapy with methotrexate was also introduced, aiming for a diseasemodifying effect, although his was a very early case of SSc. Certainly, the long-term outcomes in this case are expected to be those of severely progressive disease, due to the burden of peripheral vasculopathy as well as to the unpredictable disease course of SSc. Awareness of this rather bleak prognosis and early aggressive treatment and close follow-up are thus mandatory.

\section{CONCLUSIONS}

Critical digital ischemia remains a rare and potentially serious complication of peripheral vasculopathy in SSc. Successful short as well as long term outcomes may arise from extensive assessment, early diagnosis and prompt therapeutic intervention. However, current treatment options are far from ideal, indicating the need for a complex approach focused on pathobiologic issues. 


\section{REFERENCES}

1. Matucci-Cerinic M., Kahaleh B., Wigley F.M. Review: evidence that systemic sclerosis is a vascular disease. Arthritis Rheum 2013; 65:1953-1962.

2. Cutolo M., Sulli A., Smith V. Assessing microvascular changes in systemic sclerosis diagnosis and management. Nat.Rev. Rheumatol. 2010; 6:578-587.

3. Herrick A.L. Management of Raynaud's phenomenon and digital ischaemia. Curr. Rheumatol. Reports 2013; 15:303.

4. Minier T., Guiducci S., Bellando-Randone S., et al. Preliminary analysis of the very early diagnosis of systemic sclerosis (VEDOSS) EUSTAR multicenter study: evidence for puffy fingers as a pivotal sign for suspicion of systemic sclerosis. Ann. Rheum. Dis. 2014; 73:2087-2093.

5. Valenzuela A., Adeduntan R., Chung L. Management of Digital Ischemia. In: Wigley FM, Herrick AL, Flavahan (eds.) Raynaud's Phenomenon - A Guide to Pathogenesis and Treatment. Springer New York; 2014:339-360.

6. Sharp C.A., Akram Q., Hughes M., et al. Differential diagnosis of critical digital ischemia in systemic sclerosis: Report of five cases and review of the literature. Semin. Arthritis Rheum. 2016: article in press.

7. Marvi U., Chung I. Digital Ischemic Loss in Systemic Sclerosis. Int. J. Rheum. 2010;1-7.

8. Park J.H., Sung Y.K., Bae S.C., et al. Ulnar artery vasculopathy in systemic sclerosis. Rheumatol. Int. 2009; 29:1081-1086.

9. Raijmakers R., Renz M., Wiemann C., et al. PM-Scl-75 is the main autoantigen in patients with the polymyositis/scleroderma overlap syndrome, Arthritis Rheum. 2004; 50:565-569.

10. Hanke K., Bruckner C.S., Dahnrich C., et al. Antibodies against $\mathrm{PM} / \mathrm{Scl}-75$ and PM/Scl-100 are independent markers for different subsets of systemic sclerosis patients, Arthritis Res. Ther. 2009; 11:R22.

11. Kowal-Bielecka O., Landewe R., Avouac J. et al. EULAR recommendations for the treatment of systemic sclerosis: a report from the EULAR Scleroderma Trials and Research group (EUSTAR). Ann. Rheum. Dis. 2009; 68:620-628.

12. Hughes M., Ong V., Anderson M., et al. Consensus best practice path way of the UK Scleroderma Study Group: digital vasculopathy in systemic sclerosis. Rheumat. (Oxford) 2015;54:2015-2024.

13. Wigley F.M., Wise R.A., Seibold J.R., et al. Intravenous iloprost infusion in patients with Raynaud phenomenon secondary to systemic sclerosis. A multicenter, placebo-controlled, double-blind study. Ann. Intern. Med. 1994;120:199-206.

14. Pope J., Fenlon D., Thompson A. et al. lloprost and cisaprost for Raynaud's phenomenon in progressive systemic sclerosis. Cochrane Database Syst Rev 2000; (2):CD000953.

15. Wigley F., Herrick A. Management of Raynaud's phenomenon and digital ulcers, Current Treatments Options in Rheum 2015; 1:68-81.

16. Schiopu E., Impens A.J., Philllipset K. Digital ischemia in scleroderma spectrum of diseases, Int. J. Rheum. 2010; vol 2010, 1-8.

17. Abou-Raya A., Abou-Raya S., Helmii M. Statins: potentially useful therapy of systemic sclerosis-related Raynaud's phenomenon and digital ulcers. J Rheumatol 2008; 35:1801-8.

18. Momeni A., Sorice S.C., Valenzuela A.,Fiorentino D.F., Chung L., Chang J. Surgical treatment of systemic sclerosis-is it justified to offer peripheral sympathectomy earlier in the disease process? Microsurgery 2015; 35:441-6. 